\title{
Tracking Quantum Effects at the Nanometer Scale with EELS and Cathodoluminescence
}

\author{
M. Kociak ${ }^{1 *}$, L. H. Galvao-Tizei ${ }^{1}$, H. Lourenço-Martins ${ }^{1}$, A. Campos ${ }^{1}$, S. Meuret ${ }^{1}$, R. Bourrellier ${ }^{1}$, A. \\ Zobelli $^{1}$, M. Hillenkamp ${ }^{2}$, J. D. Blazit ${ }^{1}$, M. Tencé ${ }^{1}$ and O. Stéphan ${ }^{1}$ \\ 1. Laboratoire de Physique des solides, CNRS/Université Paris Sud, Orsay France. \\ 2. Institut Lumière Matière, CNRS/Université de Lyon 1, Villeurbanne, France. \\ * Corresponding author: Mathieu.kociak@u-psud.fr
}

In this talk, I will review recent work using EELS and cathodoluminescence that reveal the quantum nature of the quantum materials - in line with the subject of the symposium, or that make use of the quantum properties of the probe electrons. Indeed, quantum effects naturally occur when studying the optical properties of small objects. Less obviously, the quantum nature of the electron can be harnessed to unveil information usually missed when performing EELS, such as the polarization of nanoobjects.

I will first investigate the possibilities offered by cathodoluminescence to study the true quantum nature of light emission from point defects. Such point defects, when they appear in wide band gap semiconductors, are emitting in the visible-UV range. As a point defect is an atomic-like system, it can be seen essentially as a two-level system, and therefore acts as a single-photon emitter (SPE). SPEs are central building blocks of quantum nanooptics, but are so small that their structure cannot be accessed without the use of electron microscopy techniques. Adding quantum nanooptics detection strategy in cathodoluminescence, as we did for nitrogen-vacancy in diamond [1] or UV emitters in hBN [2] seems therefore essential.

Next, I will discuss the measurement of surface plasmons in metallic clusters by EELS. In these clusters, a large variety of effects, including purely quantum ones like confinement or spill-out, influence the plasmon resonance energy and damping. EELS studies of metallic clusters has been the subject of a controversy [3], because the EELS data [4] and related theory could not explain observed the discrepancies with the vast literature on metallic cluster studied by macroscopic optical means. We have recently shown [5], combining optical and EELS experiments, and building a minimal theory valid for both techniques, that the discrepancies could be easily understood as related to variations in samples and the effect of the environment which is much more important in the quantum regime reached by plasmons in clusters than in the regular classical limit.

Finally, I will introduce new theoretical developments taking fully into account the quantum nature of the incoming and outgoing waves as used in an EELS experiment. I will show how the quantum nature of the electron can be used to tackle non-trivial physical questions, such as the nature of super-chiral fields. If time allows, I will discuss the limitation of those theories and related experiments to fully describe the quantum nature plasmons or excitons in nanostructures.

References:

[1] Tizei, L. H. G. \& Kociak, M. Phys. Rev. Lett. 110 (2013), p. 153604.

[2] Bourrellier, R. et al., Nano Lett. 16 (2016), p. 4317. 
[3] Haberland, H. BRIEF COMMUNICATIONS ARISING. Nature Publishing Group 494 (2013), p. E1.

[4] Scholl, J. A., Koh, A. L. \& Dionne, J. A. Nature 483 (2012), p. 421.

[5] Campos, A. et al., Nat Phys 111 (2018), p. 1.

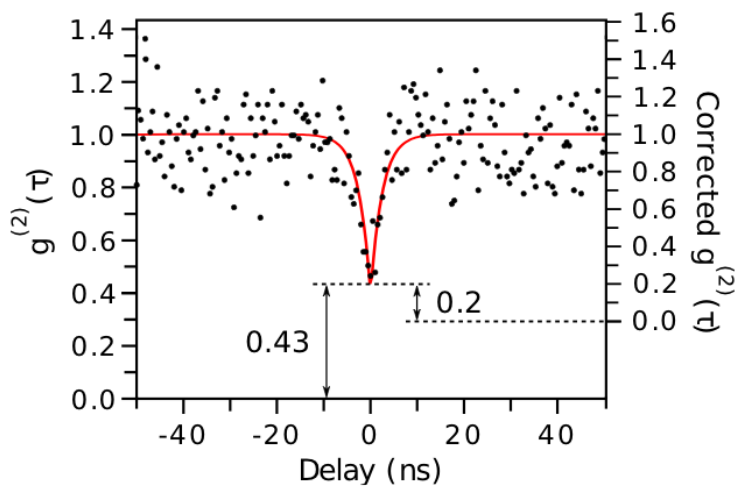

Figure 1. Cathodoluminescence time-auto-correlation function from a UV-emitting point defect in $\mathrm{hBN}$. The dip at zero-delay is typical from an antibunching behavior, a signature of single photon emission. After [2].
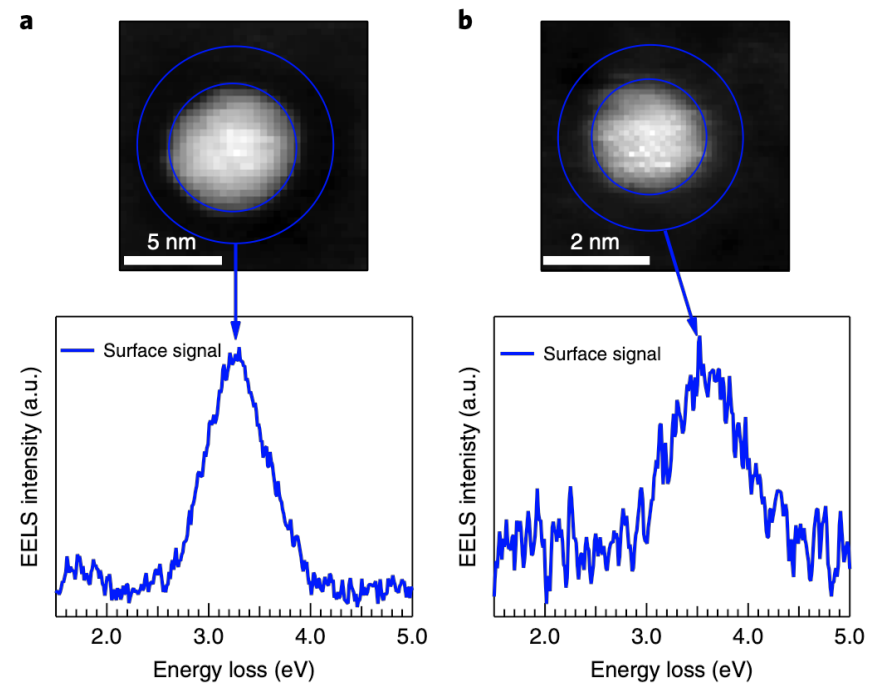

Figure 2. EELS signal coming from the surface of silver nanoclusters embedded in silicon oxide. The observed blue shift could be naively attributed to a quantum confined effect as the particle size decreases. A full quantum treatment shows that spill-out and quantum confinement should cancel due to the presence of the matrix, and no blue-shift is expected; this shift is most likely due to the change in matrix porosity due to electron irradiation. After [5]. 